\title{
STUDIES IN ARTIFICIAL PARTHENOGENESIS
}

\author{
IV. HEAT PARTHENOGENESIS
}

L. V. HEILBRUNN

University of Michigan

Earlier study has shown that all agents which cause artificial parthenogenesis in the sea-urchin egg produce a coagulation in the cytoplasm (Heilbrunn, '15). This does not mean that all types of coagulative change cause the egg to begin its development, for, as is fairly obvious, some coagulations cause only death.

It is well known that other eggs differ from the sea-urchin egg in their susceptibility to various parthenogenetic agents, and it is of interest to study these eggs and to compare them with each other and with the sea-urchin egg. It is of particular importance to include eggs in different stages of maturation. The sea-urchin egg is already mature when it is shed into the sea-water, but many eggs at the time of fertilization have not completed the first maturation division and still retain the first maturation spindle. The behavior of these eggs is particularly interesting, for they already have a spindle, and it is difficult to understand what effect a coagulation might have upon them. Other eggs are in the germinal vesicle stage at the time the sperm enters, and yet they, too, may be made to start their development artificially.

In the second paper of this series I tested the effect of all parthenogenetic agents on one egg. In the present study I have attempted to determine the effect of a single parthenogenetic agent on several types of eggs.

Of all parthenogenetic agents heat is perhaps the most generally applicable. Heat parthenogenesis has been described 
for the starfish egg by Delage ('01), R. S. Lillie ('08, '15); for Cumingia by Morris ('17), for Nereis by Just ('15), and for Chaetopterus by Allyn ('12). Heat is the best treatment known for the starfish, Nereis, and Chaetopterus eggs, and in these forms it gives surprisingly good results.

So far no one has furnished any good evidence as to what the heat does to the egg. It is true that a rise in temperature causes an increase in oxidations, but this increase is slight, a $10^{\circ}$ rise in temperature eausing only a twofold increase in oxidations (Loeb and Wasteneys, '11). According to R. S. Lillie ('15), the temperature coefficient for heat parthenogenesis is several hundred for each $10^{\circ}$ rise in temperature. We can scarcely assume, therefore, that heat produces its effect merely by increasing the rate of some chemical reaction, for in general chemical reactions have a very low temperature coefficient. Lillie points out that the heat must have some physical effect, and he believes this to be a liquefaction. In favor of this theory he cites no direct evidence, and the high temperature coefficient which he describes could be explained just as well by a gelation or coagulation as by a liquefaction. Chick and Martin ('10) have shown high temperature coefficients in the coagulation of egg albumen, and I have recently shown a high temperature coefficient in the heat coagulation of protoplasm ('24). Another theoretical interpretation is that of Just ('15). He believes that heat has some effect on the hypothetical substance fertilizin, for he finds that after several washings in sea-water Nereis eggs. no longer respond to heat treatment.

In this study I have endeavored to find out whether the temperatures used in heat parthenogenesis have any observable effect on the physical consistency of the egg cytoplasm. The work was done at the Woods Hole Marine Biological Laboratory during the summer of 1923 . Three types of eggs were used, those of the sea-urchin Arbacia, of the annelid Nereis, and of the clam Cumingia. The Arbacia egg is fully mature when shed, the Nereis egg has not yet begun its maturation divisions, and the Cumingia egg has a fully formed spindle of the first maturation division. 
In heating the egg's constant temperatures were maintained with the aid of a de Khotinsky constant-temperature apparatus. A test-tube containing sea-water was placed in the apparatus and brought up to the desired temperature. The eggs were then added in a few drops of sea-water. In most cases the temperature remained constant within a tenth of a degree, although in some cases it varied slightly more than this.

In testing the effect of heat on protoplasmic consistency, the centrifuge method of measuring viscosity was used. The technique of this method has been fully described in earlier papers (Heilbrunn, '20 a, '21). In the present study a Bausch \& Lomb hand centrifuge was used. When the high-speed handle of this instrument is turned once per second, a force 4968 times gravity is obtained.

In discussing the experimental results, I shall consider the case of each kind of egg separately.

\section{ARBACIA}

There has been no real description of heat parthenogenesis in the sea-urchin egg. At any rate, Harvey ('10), in his compilation of all the methods used in obtaining artificial parthenogenesis, cites no case of heat being used successfully on this egg. The only record I have found is a bare mention by McClendon ('10) that he kept Arbacia eggs at a temperature of $32^{\circ}$ for four minutes and observed segmentation. Apparently he did only one experiment and he does not give the per cent of segmentation that he observed.

When a sea-urchin egg is heated it does not ordinarily undergo any visible cortical change. This fact, already noted in an earlier paper ('15), is perhaps the reason that heat is so inefficient in producing segmentation in this egg. In several experiments, however, after prolonged heat treatment small blister-like processes could be seen on the surface of some of the eggs. Although this 'blister formation' was not carefully studied, it is apparently associated with a pronounced coagulation of the cytoplasm. Such a coagulation 
would cause the egg contents to pull away from the vitelline membrane at various points.

Heat parthenogenesis does occur in the sea-urchin egg, although the percentages of segmenting eggs obtained with this method are never very high. In order to have any effect the heat must act on the eggs long enough to produce a coagulation in the eytoplasm. This is shown by the following experiments.

July 15th. Arbacia eggs were placed in sea-water at a temperature of $32.3^{\circ}$ at $11: 12$ A.M. The heated eggs were tested at $11: 17$ and again at 11:22 A.M., the high-speed centrifuge handle being turned 40 times in 40 seconds. In these tests the zones showed plainly. A similar test at $11: 27 \frac{1}{2}$ A.M. showed the hyaline zone only faintly, and another test five minutes later, at $11: 32 \frac{1}{2}$ A.M., failed to show a hyaline zone, although the centrifugal treatment was the same as in the earlier tests. Thus in this experiment coagulation occurred within about 20 minutes. Eggs were removed from the hot sea-water at $11: 32 \frac{1}{4}$ A.M., at $11: 39$ A.M., and at $11: 45$ A.M., and were placed in cool sea-water in Stender dishes A, B, and C, respectively. Counts of segmenting eggs were made several hours later. No cleavage could be found in dishes $\mathrm{A}$ and $\mathrm{B}$, but in $\mathrm{C}$, which contained eggs exposed 33 minutes, 17 segmented eggs were found out of a total of 500 which were counted.

July 16th. At 10:31 A.M., Arbacia eggs were placed in sea-water at a temperature of $32.9^{\circ}$. They were transferred from the hot sea-water to cool sea-water in Stender dishes A, B, C, D, E, F, G, H, after exposures of $5,10,15,20,25,30,35,45$ minutes, respectively. Centrifuge tests made as in the previous experiment showed that coagulation occurred at about 15 minutes after the eggs were exposed to the heat. At about $2: 15$ P.M., examination of Stender dishes $\mathrm{A}-\mathrm{H}$ gave counts as listed below. In the fractions the numerator indicates the number of segmenting eggs, the denominator the total number of eggs counted.

$\begin{array}{llllllllll}\text { Exposure-minutes } & \ldots \ldots \ldots \ldots & 5 & 10 & 15 & 20 & 25 & 30 & 35 & 45\end{array}$

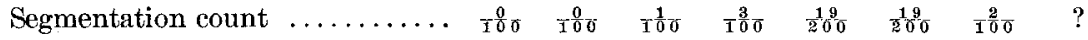

In the eggs exposed to the heat 35 and 45 minutes, fragmentation had begun, and these counts were therefore difficult to make.

July 17th. The temperature of the warm sea-water was $31.5^{\circ}$. At this temperature the time necessary for coagulation to take place

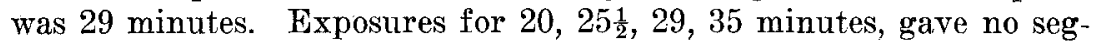
mentation. An exposure of 45 minutes showed some eggs with 
cleavage furrows indicated, but of a hundred eggs exposed for this length of time none showed certain cleavage. An exposure of 55 minutes resulted in 4 per cent cleavage. In addition, a number of other eggs showed fragmentation.

July 21st. Eggs were exposed to a temperature of $32.2^{\circ}$ for 31 , $41,52,66$, and 87 minutes. At this temperature the time necessary for coagulation was found to be 24 minutes. Segmentation counts several hours later gave the following results:

$\begin{array}{lllllll}\text { Exposure-minutes } & \ldots \ldots \ldots \ldots & 31 & 41 & 52 & 66 & 87\end{array}$

Segmentation count $\ldots \ldots \ldots \ldots . \quad \frac{1}{10} \overline{0} \quad \frac{6}{10} \overline{0} \quad \frac{9}{10} \overline{0} \quad \frac{15}{100} \quad \frac{1}{10} \overline{0}$

The count for the 66-minute exposure is somewhat doubtful, for the dish containing the eggs was full either of isolated blastomeres or of fragmented portions of the eggs.

These experiments show that the very temperatures which are effective in evoking artificial parthenogenesis cause a coagulative change in the cytoplasm of the egg. Furthermore, it has been shown that only those exposures to heat which cause coagulation result in any initiation of development. The sea-urchin egg must actually suffer this coagulative change for some few minutes if the heat treatment is to be successful. Of course, too prolonged a heat coagulation causes not development, but death.

\section{NEREIS}

Just ('15) has performed some very careful experiments on heat parthenogenesis in the egg of Nereis, and his experiments furnished the starting-point for my own investigation. His results are easily confirmed, and heat appears to be by far the most reliable method for producing artificial parthenogenesis in this egg. Just found that a temperature of $33^{\circ}$ to $35^{\circ}$ caused initiation of development, and this development was preceded by a normal cortical change involving a typical extrusion of jelly. As to the nature of the effect that heat has on the egg Just has very little to say. He finds that two or three washings of the eggs in sea-water will effectively prevent heat parthenogenesis, and he interprets this fact on the basis of the fertilizin hypothesis. He believes 
that the washings remove fertilizin from the egg, and it is this lack of fertilizin that prevents the heat from having its effect. Heat acts, says Just, in the same manner as the sperm, "through the activation and the binding of the fertilizin."

This interpretation of Just's is somewhat indefinite, for fertilizin is not a known substance and the term activation is not used in any precise sense. As a matter of fact, washed Nereis eggs, supposedly free from fertilizin, can be restored to their original heat-sensitive condition by the addition of a slight amount of acid to the sea-water in which they are contained. In two experiments this was accomplished by the addition of $0.2 \mathrm{cc}$. of $\mathrm{n} / 10 \mathrm{HCl}$ to $10 \mathrm{cc}$. of sea-water containing the washed eggs. In another experiment this concentration did not give the desired result, but in this case the eggs were restored to their original sensitive condition by immersion for a minute in $10 \mathrm{cc}$. sea-water plus $0.4 \mathrm{cc} . \mathrm{n} / 10$ $\mathrm{HCl}$. Although only three experiments were performed, it seems probable that a slight amount of acid is all that is necessary to make washed eggs sensitive to heat again. It thus seems likely that unwashed eggs differ from washed eggs in being surrounded by, or in containing, a fairly high concentration of carbon dioxide. If washing of the Nereis egg deprives it of its essential fertilizin, then it would appear from these facts that fertilizin in this instance is merely carbon dioxide. This is perhaps the true explanation of Just's results, but it does not bring us much closer to an understanding of heat parthenogenesis.

Our main interest is an attempt to show that heat treatment such as that used by Just causes coagulative changes in the protoplasm of the Nereis egg. The experiments very decisively favor this view.

It must be remembered that the Nereis egg is in the germinal-vesicle stage when ready for fertilization. The effect of the heat is first to cause an extrusion of jelly, which is accompanied in the interior of the egg by the breaking down of the germinal vesicle. When the germinal vesicle breaks down the protoplasm becomes liquefied, and this is the first 
effect of the heat. A similar change occurs after insemination (cf. Heilbrunn, '21). When the eggs remain in the warmth the liquefaction is followed by a coagulation. If the eggs are to develop parthenogenetically, they must remain in the warm sea-water until this coagulation has occurred.

July 28th. At 11:12 A.M., some freshly shed eggs were placed in a test-tube containing warm sea-water at a temperature of $33^{\circ}$. At various intervals the eggs were centrifuged, in every case the centrifugal treatment consisting of 5 turns of the high-speed handle in 5 seconds. At 11:16 A.m., the jelly had already been extruded, and the germinal vesicle had apparently begun to break down, but when the eggs were centrifuged at this time they showed no zones. When they were centrifuged at $11: 23$ A.M., after an exposure of 11 minutes and following the complete breakdown of the germinal vesicle, a hyaline zone appeared through a third of the egg. After a 15-minute exposure, at 11:27 A.M., centrifugal treatment showed a hyaline zone, but it did not appear to be as wide as in the previous test. After 19 minutes' exposure about half the eggs failed to show a hyaline zone on being centrifuged in the usual manner, and after 25 minutes only a few showed a very narrow hyaline zone, and the great majority of the eggs showed no hyaline zone at all. Thus coagulative changes were certainly present after an exposure of the eggs to a temperature of $33^{\circ}$ for 19 minutes.

Eggs were removed from the warm sea-water following exposures of $4,11,15,19,25,31,38,49$ minutes. The eggs exposed 4, 11, 15 minutes showed no segmentation. In the 19-minute exposure, one egg segmented out of 100 examined. No segmentation was observed in the 25-minute exposure. The 31-, 38-, and 49-minute exposures showed 4, 10, and 12 per eent of segmenting eggs at 2 P.M., and 6,18 , and 46 per cent of segmenting eggs 2 hours later.

August 4th. Some freshly shed eggs were placed in $10 \mathrm{cc}$. of seawater at a temperature of $34.9^{\circ}$. The eggs were exposed to the heat for $8,13 \frac{1}{2}, 18,23,28$ minutes, and were then transferred to seawater at room temperature. Centrifuge tests were made as in the previous experiment, in each case the high-speed handle being turned 5 times in 5 seconds. A test after 8 minutes' exposure showed a hyaline zone extending through a third of the egg. After $13 \frac{1}{2} \mathrm{~min}$ utes' exposure, the eggs when centrifnged in the usual manner generally failed to show a hyaline zone, although this zone appeared in a few eggs. A test after 18 minutes showed no zones generally. Segmentation counts were made about 3 hours later. The 8-minute exposure resulted in no segmentation, the $13 \frac{1}{2}$-minute exposure gave 
1 per cent of segmenting eggs, the 18-minute exposure gave 4 per cent, the 23 -minute exposure 37 per cent, and the 28 -minute exposure 12 per cent.

In various other experiments similar results were obtained. It is certain that in every case the heat causes a coagulation of the cytoplasm. Those exposures to heat which are too short to produce any coagulation never result in segmentation of the egg. In heat parthenogenesis in Nereis the relation between coagulation and initiation of development is definite and exact.

\section{CUMINGIA}

The sea-urchin egg when ready for fertilization has completed its maturation divisions. In the Nereis egg the maturation divisions have not yet begun when the egg is shed into the sea-water. A third case is presented by the Cumingia egg. When this egg is shed it has its first maturation spindle fully developed, but it is nevertheless in a resting stage, and unless fertilization occurs it remains in the metaphase of the first maturation division. The block which prevents completion of this division is the restraint imposed by the stiff vitelline membrane. As was shown several years ago (Heilbrunn, ' $20 \mathrm{~b}$ ), any method of removing this restraint results in a completion of maturation. The membrane may be softened by swelling, it may be lifted from the egg or shaken off; in any case, the maturation divisions proceed to their conclusion. But when maturation is made to proceed in this way no segmentation follows, and it is evident that the maturation spindle is not of its own accord able to transform itself into a cleavage spindle. Some other force is necessary, either to transform the maturation spindle into a cleavage spindle or to originate a new cleavage spindle.

In her study of artificial parthenogenesis in Cumingia, Morris ('17) showed that development could very successfully be induced by a heat treatment followed by a treatment with hypertonic sea-water. She was interested primarily in obtaining a good method of artificial parthenogenesis so 
that she might make a cytological study, and she did not attempt to discover the nature of the heat effect. Moreover, she combined the action of heat and of hypertonic solutions. This doubtless gives better results in parthenogenesis, but the combination of treatments does not aid in our analysis of the nature of the heat effect.

Morris found that the best temperatures for the artificial parthenogenesis were from $32^{\circ}$ to $37^{\circ}$. These temperatures cause coagulative changes in the egg, as has been shown in a recent paper (Heilbrunn, '24). They probably also cause a slight membrane swelling, but this subject has not been thoroughly investigated.

In an early experiment it was found that eggs exposed to a temperature of $33^{\circ}$ for sixty minutes showed 54 per cent of segmentation. In these eggs coagulative changes occurred after fifty-three minutes' exposure to the warm temperature. Although an unusually high percentage of eggs segmented in this experiment, there was practically no polar body formation. This is in accord with Morris' observation that longer exposures at lower temperatures favor polar body formation rather than cleavage. Additional evidence was obtained in support of this view, and it seems probable that the tendency for polar body formation and for cleavage tend to exclude each other. Higher temperatures give larger percentages of polar body formation and smaller percentages of segmentation. Thus at a temperature of $37.5^{\circ}$, exposures of 2 , $4,8,17$ minutes gave $0,1,5$, and 0 per cent cleavage and $12,26,18$, and 0 per cent polar body formation. Apparently longer exposures favor cleavage. In an experiment at $35.1^{\circ}$ the per cent of cleavage increased with the length of exposure, whereas the per cent of polar body formation decreased. This is shown in the following table. In this table the polar body count for the twenty-three-minute exposure is not very exact, for in the longer exposures the polar bodies became large and it was hard to distinguish them from blastomeres. 


$\begin{array}{ccc}\begin{array}{c}\text { Expostre- } \\ \text { minutes }\end{array} & \begin{array}{c}\text { Percent } \\ \text { cleavage }\end{array} & \begin{array}{c}\text { Per cent } \\ \text { polar body formation }\end{array} \\ 2 & 0 & 5 \\ 4 & 0 & 4 \\ 6 & 0 & 5 \\ 10 & 5 & 4 \\ 23 & 16 & 3 \\ 31 & 25 & 0\end{array}$

The facts apparently favor the view that, in some instances at least, the maturation spindle may in one way or another be converted into a cleavage spindle. This is a point of considerable importance and it will be referred to again.

The question now arises if coagulation can be shown to precede heat parthenogenesis in every case. The facts are not as clear cut in Cumingia as they are in Arbacia or in Nereis, and for that reason it will be necessary to cite a larger body of evidence. Unfortunately, some of the experiments were performed in the latter part of August, and although the eggs are fertilizable and can be made to develop parthenogenetically at this time, they are much more variable than they are earlier in the season, and sometimes they behave very queerly. Oftentimes they show a tendency to become flattened, and when they assume this flattened shape comparative viscosity tests are not as reliable as they would be if the eggs remained spherical. For conditions of movement of granules probably become changed as the eggs flatten, and certainly conditions of observation vary, for it is of course easier to see a hyaline zone in a flat egg than in a spherical egg.

In a recent paper a curve of heat coagulation has been plotted for the protoplasm of the Cumingia egg (Heilbrunn, '24). This curve gives the time in minutes at which coagulation occurs at various temperatures. In making the tests for this curve the eggs were centrifuged for five seconds at the usual speed of one turn of the high-speed handle per second. Ordinarily the eggs show zones after two seconds of centrifugal treatment, if they fail to show zones after five seconds, this indicates at least a threefold increase in proto- 
plasmic viscosity. The curve that has been plotted therefore gives the time necessary at each temperature for a threefold increase in viscosity. If the eggs fail to show zones after three seconds of centrifugal treatment, this indicates at least a twofold increase in viseosity. Such a three-second test was used in some of the experiments.

August 14th. At 2:40 P.M., some Cumingia eggs were placed at a temperature of $34^{\circ}$. They were centrifuged at various intervals for three seconds at the usual speed. A test after 4 minutes' exposure showed zones. After 6 minutes most eggs showed zones following centrifugal treatment, but a few eggs lacked them. After $7 \frac{1}{2}$ minutes no zones appeared generally as a result of the centrifugal treatment, although a few eggs showed zones faintly. Eggs were removed from the warmth after exposures of $4,6,7 \frac{1}{2}, 11,16,21,30$ minutes. The first two exposures showed no cleavage, the $7 \frac{1}{2}$-minute exposure showed $1 \frac{1}{2}$ per cent, the 11 minute exposure 17 per cent, the 16-minute exposure 22 per cent, and the other two exposures $11 \frac{1}{2}$ and 25 per cent cleavage, respectively.

At 3:30 P.M., another lot of eggs was placed at a temperature of $34.6^{\circ}$. Centrifuge tests were made as before. As a result of these tests, zones were found generally present after 3 minutes' exposure, but after $4 \frac{1}{2}$ minutes the zones were either faint or lacking, the eggs being about evenly divided in this respect. At $5 \frac{1}{2}$ minutes the zones were lacking in a large majority of the eggs, and a minute later there were no zones generally. Of course, these tests had to be performed in great haste and are not as reliable as they might have been if they could have been made more slowly. Eggs were removed from the warm sea-water after $3,7,12,20,30,40,50$ minutes, and segmentation counts for these various exposures gave $0.5,3.5,9,12,18,18$, and 16 per cent, respectively. In the two longest exposures many of the eggs were broken up.

August 23rd. At 3:55 P.M., eggs were placed at a temperature of $35.1^{\circ}$. At various times they were centrifuged for two seconds at the usual speed. Such a centrifugal treatment is barely sufficient to show zones in normal untreated eggs at room temperature, and if zones do not appear, it indicates only a slight increase in viscosity. Eggs centrifuged after a 2-minute exposure showed zones generally. After 4 minutes the eggs still showed zones on being centrifuged, at this time some of the eggs were already beginning to flatten. After 6 minutes zones appeared generally after centrifuging, they showed more plainly in the flatter eggs. After 10 minutes' exposure the heated eggs were centrifuged simultaneously with the control of 
untreated eggs. When the two sets of eggs were compared, the heated eggs showed either no zones or the dark zone only in the flatter eggs, whereas the control eggs showed the dark zone in all cases, although the pigment zone was indistinct. The heated eggs were again centrifuged after 23 minutes' exposure. At this time some eggs showed zones and others lacked them, when the zones appeared it was in the flatter eggs. After a 31-minute exposure no zones appeared after centrifugal treatment except in a few rare instances. Eggs were removed from the heat after exposures of $2,4,6,10,23,31$ minutes. No segmentation occurred in the first three exposures, the last three gave 5 per cent, 16 per cent, and 25 per cent, respectively.

August 29th. At 12:06 P.M., eggs were placed at a temperature of $34.5^{\circ}$. Eight minutes later the eggs were centrifuged for 2 seconds. Zones appeared generally. At this time the eggs were already beginning to flatten. After 10 minutes' exposure another test of the same sort also showed zones. The eggs by this time were rather flat. After a 13-minute exposure a 3 -second centrifuge test showed zones in nearly all the eggs, but there were a few exceptional eggs which did not show zones. After 16 minutes a 2-second test showed zones in most of the eggs, but a few eggs lacked them. After 18 minutes a similar test showed most eggs without zones, and after 21 minutes a centrifuge test for 5 seconds showed most of the eggs without zones. The eggs were returned to sea-water at room temperature after exposures of 4, 8, 13, 18, 23, 27 minutes. The 4-minute exposure showed no segmentation and the 8-minute exposure showed only one segmenting egg out of a hundred examined. The other four exposures showed $8,33,26$, and 30 per cent segmentation, respectively.

Other experiments of the same sort could be cited. In general it appears to be true that coagulation occurs in the warmth before the egg is stimulated to begin its development. Eggs which have been exposed to higher temperatures for so short a period that coagulation has not had time to occur do not ordinarily start their development. In the experiments cited, two exceptions occur. In the second experiment of August 14th, 0.5 per cent of the eggs segmented after a three-minute exposure-an exposure not long enough to provoke coagulation in the eggs which were tested. This case involves a single egg - one out of two hundred. In view of the fact that in this experiment coagulation began after four and a half minutes' exposure, it is not much of an assumption to suppose that the exceptional egg became 
coagulated slightly in advance of the rest. Another exceptional egg occurred in the eight-minute exposure of the experiment of August 29th. The same explanation probably holds for this case. Late in the season the eggs are generally more variable, and it is easy to understand how now and then an egg may be coagulated before the rest of the lot.

But even if we grant this explanation of the exceptional cases, it is obvious that in the Cumingia egg heat coagulation does not always precede initiation of development by the marked time interval observed in the eggs of Arbacia and Nereis. It is true that the highest percentages of segmentation are obtained when the heat has acted for quite a few minutes longer than necessary to cause a pronounced coagulation. But there are usually some eggs which begin to segment after a heat treatment barely long enough to cause any coagulation at all. What is the reason for this difference in the case of Cumingia? The answer seems clear. In Arbacia and Nereis heat coagulation produces a spindle, and this effect takes time. Perhaps in many instances heat also produces a new spindle in the Cumingia egg. But this is probably not always the case, for the Cumingia egg has a fully formed spindle when it is shed into the sea-water. In order to induce cleavage in this egg, it is not necessary to create a spindle, but only to transform the maturation spindle into a cleavage spindle. This apparently does not require as great a coagulation in the cytoplasm. Such an explanation need not be assumed for all the eggs exposed to heat. Cumingia eggs behave in a variety of ways and not all of them go through the same processes. Some eggs give off polar bodies before segmenting, as Morris ('17) has pointed out, and some apparently begin maturation, and then segment before the maturation process is completed.

In support of the view that heat in some instances transforms the maturation spindle into a cleavage spindle, it should be recalled that both Morris and myself found that those heat treatments which gave higher percentages of polar body formation tended to give lower percentages of segmentation, and vice versa. 
Finally, it may be asked how a coagulation could conceivably change a maturation spindle into a cleavage spindle. Earlier work on cell division has shown that the astral rays are fastened to the outer membrane of the cell (Conklin, '17; Heilbrunn, '17, '20). Probably in a maturation spindle nearly all of the rays are fastened to the animal pole or its vicinity, and this is why the maturation results in the cutting off of a very small cell. If the whole cell is to be divided, then it is necessary for the spindle to be attached to both poles of the egg. It seems likely that even a slight coagulation could complete this attachment. Only a small coagulative change would be necessary to convert a maturation spindle into a cleavage spindle.

This point of view is supported by the fact that in some instances, when the eggs are treated with heat they tend to flatten. Such flattening is a very common occurrence late in the season, and for a long time it was much of a mystery. I was at a loss to understand the causes which might underlie such a process. At last I was led to assume that it was due to a shortening which followed the anchoring of the spindle at both poles of the egg. This might result in a tension across the polar axis, and if the vitelline membrane were weak enough, the egg would flatten. If this explanation is correct, then in the flat, dise-like eggs, the axis of the spindle should be at right angles to the disc. Observation of flattened eggs indicated this to be true, although the angle between the disc and the spindle is not always an exact right angle.

Although the experiments with Cumingia involve more detailed explanation, it is obvious that they furnish corroborative evidence in favor of the main thesis. In Cumingia, too, heat parthenogenesis is regularly preceded by coagulation. This coagulation may cause the formation of a spindle, or it may convert the maturation spindle already present in the egg into a cleavage spindle. 


\section{DISCUSSION}

Although heat is one of the most general methods of inducing artificial parthenogenesis, no one has ever brought together any direct evidence to show how it affects the egg. It has never been demonstrated that the temperatures which cause the egg to begin its development have any particular effect either on its permeability or on its oxidation rate. This investigation has shown that at the temperatures and exposures used to induce artificial parthenogenesis a measurable coagulation occurs. The facts lend strong support to the viscosity or coagulation theory.

This theory claims that all the methods which incite the egg to segmentation produce a gelation or coagulation within the egg. It is a theory easily capable of test. To oppose it one has only to show that some effective method of artificial parthenogenesis causes no gelation or coagulation. So far the evidence has been all in favor of the theory. In the seaurchin egg all the agents which cause the egg to segment can by actual test be shown to produce a gelation or coagulation. In my first statement of the theory (Heilbrunn, '15), I grouped these agents in two classes, those which caused exosmosis and those which caused endosmosis. The latter class included reagents like ether and chloroform. This classification is not an advantageous one. Some agents cause coagulation of the protoplasm quite irrespective of their osmotic effect on the cell. In many instances the coagulative change is soon followed by death, and there is a very narrow margin between the amount of coagulation which will start the egg to develop and the amount of coagulation which will produce death. When eggs are subjected to heat, a moderate exposure causes a coagulation which results in artificial parthenogenesis, a slightly longer exposure causes an irreversible coagulation which is death.

The experimental results of this investigation offer additional support to the coagulation theory. It has been shown clearly for three different types of eggs that coagulation in all cases precedes initiation of development by heat. In the 
Cumingia egg, which already has a spindle when it is shed, not so marked or so prolonged a coagulation is necessary. In this case, apparently, only enough coagulation is needed to convert the maturation spindle into a cleavage spindle.

The actual demonstration of coagulative change in all sorts of heat parthenogenesis as well as in other sorts of artificial parthenogenesis places the coagulation theory on a different footing from the other theories which have been advanced to explain the process. These theories have merely postulated changes of one sort or another, they have never shown these changes to occur in any wide range of cases.

It should be remembered that the coagulation theory does not assume a total coagulation of the egg. Only certain constituents, or perhaps only a single constituent is responsible for the production of the mitotic spindle.

Nor should it be supposed that all types of coagulative change are essentially the same. It is believed that heat coagulation and coagulation by salt solutions are distinctly different phenomena. The former is apparently concerned with some dissolving action on the fatty substances of the cell (Heilbrunn, '24), the latter could have no such effect. It has already been pointed out that there are two types of coagulation in protoplasm, that the coagulation by acid differs markedly from the coagulation by concentrated salt solutions (Heilbrunn, '20). Apparently heat coagulation and acid coagulation resemble each other more than they resemble coagulation by hypertonic salt solutions. This is a subject which will probably be investigated more closely in the future. It is mentioned at this point because it seems worth while to remark that some eggs undergo a more normal parthenogenetic development if treated with one class of coagulative agent rather than the other. Thus sea-urchin eggs undergo a longer and more normal development if treated with concentrated salt solutions, and this probably applies also to Cumingia eggs. On the other hand, such a method is not very effective for the eggs of Nereis or starfish. In these eggs the best development is induced either by heat, as in Nereis, or by heat or acid, as in the starfish. 


\section{SUMMARY}

In the eggs of Arbacia, Nereis, and Cumingia, temperatures which cause artificial parthenogenesis produce a coagulation within the cytoplasm of the egg. If the eggs are to be made to begin their development, they must be kept in the warmth long enough for this coagulative change to occur.

\section{LITERATURE CITED}

Alliyn, Harriett M. 1912 The initiation of development in Chaetopterus. Biol. Bull., vol. 24, pp. 21-72.

Chick, Harriette, and Martin, C. J. 1910 On the 'heat coagulation' of proteins. Jour. Physiol., vol. 40, pp. 404-430.

Conklin, E. G. 1917 Effeets of centrifugal force on the structure and development of the eggs of Crepidula. Jour. Exp. Zoöl., vol. 22, pp. $311-419$.

Delage, Y. 1901 Études expérimentales sur la maturation eytoplasmique et sur la parthénogenèse artifieielle chez les Échinodermes. Arch. de Zool. exp. et gen., 3me Ser., T. 9, pp. 285-326.

HARvey, E. N. 1910 Methods of artificial parthenogenesis. Biol. Bull., vol. 18, pp. 269-280.

HeirbrunN, L. V. 1915 Studies in artificial parthenogenesis. II. Physical changes in the egg of Arbacia. Biol. Bull., vol. 29, pp. 149-203.

1917 An experimental study of cell division. Anat. Rec., vol. 11, pp. $487-489$.

1920 a An experimental study of cell division. I. The physical conditions which determine the appearance of the spindle in sea-urchin eggs. Jour. Exp. Zoöl., vol. 30, pp. 211-237.

$1920 \mathrm{~b}$ Studies in artificial parthenogenesis. III. Cortical change and the initiation of maturation in the egg of Cumingia. Biol. Bull., vol. 38 , pp. $317-339$.

1921 Protoplasmic viscosity changes during mitosis. Jour. Exp. Zoöl., vol. 34, pp. 417-447.

1924 The colloid chemistry of protoplasm. IV. The heat coagulation of protoplasm. Amer. Jour. Physiol., vol. 69, pp. 190-199.

Just, E. E. 1915 Initiation of development in Nereis. Biol. Bull., vol. 28, pp. $1-17$.

LILLIE, R. S. 1908 Momentary elevation of temperature as a means of producing artificial parthenogenesis in starfish eggs and the conditions of its action. Jour. Exp. Zoöl., vol. 5, pp. 375-428.

1915 On the conditions of activation of unfertilized starfish eggs under the influence of high temperatures and fatty acid solutions. Biol. Bull., vol. 28, pp. 260-303.

Loeb, J., AND Wasteneys, H. 1911 Sind die Oxydationsvorgänge die unabhängige. Variabel in den Lebenserscheinungen? Biochem. Zeitsch., Bd. 36 , S. $345-356$. 
McClendon, J. F. 1910 On the dynamies of cell division. II. Changes in permeability of developing eggs to electrolytes. Amer. Jour. Physiol., vol. 27 , pp. 240-275.

MorRis, MargareT 1917 A eytological study of artificial parthenogenesis in Cumingia. Jour. Exp. Zoöl., vol. 22, pp. 1-51. 